\title{
DISRUPTION AND DAMAGE OF AN ELECTROOPTIC MODULATOR BY PULSED MICROWAVES
}

\author{
Ross T. Schermer, Frank Bucholtz, Carl A. Villarruel, Jesus Gil Gil, Tim D. Andreadis \\ and Keith J. Williams \\ U.S. Naval Research Laboratory, Washington, DC
}

\section{Introduction}

High-power microwave (HPM) sources pose a growing a threat to avionic systems [1-3]. In particular, microwave receivers that employ sensitive low-noise amplifiers (LNAs) are susceptible to damage by a high-power input. Although electronic limiters may be used to protect the LNA and subsequent receiver electronics, conventional high-power limiters can suffer from non-instantaneous turnon, relatively slow recovery, and limited power protection. An attractive alternative is to replace the electronic LNA and RF limiter with a low-noise photonic link, which eliminates all electrical connections between antenna and receiver. Key to this approach, however, is the electro-optic (EO) modulator's ability to withstand a high-power input, both in terms of permanent damage and short-term disruption. In this paper, we investigate the susceptibility of a commercial EO modulator to disruption and damage by microwave pulses. We report a $200 \mathrm{~W}$ modulator damage threshold that is on par with many high-power RF limiters. Significant disruption at peak powers as low as a few Watts, however, indicates a need for improved modulator power tolerance.

\section{Experiment and Results}

The experimental setup is shown in Fig. 1(a). Microwave pulses with $2.0 \mathrm{GHz}$ center frequency were amplified by a traveling-wave tube amplifier, and then launched directly into the coaxial RF input port of an X-cut $\mathrm{LiNbO}_{3}$ Mach-Zehnder intensity modulator. The optical transmission of the modulator was concurrently measured by transmitting light from a $1550 \mathrm{~nm}$ wavelength laser through the modulator and onto a high-frequency $(18 \mathrm{GHz})$ photodiode $(\mathrm{PD})$. Link transient response was recorded using a 20 $\mathrm{MHz}$ bandwidth oscilloscope, which functioned as a pulse envelope detector and also responded to lower frequency variations in the modulator transmission.
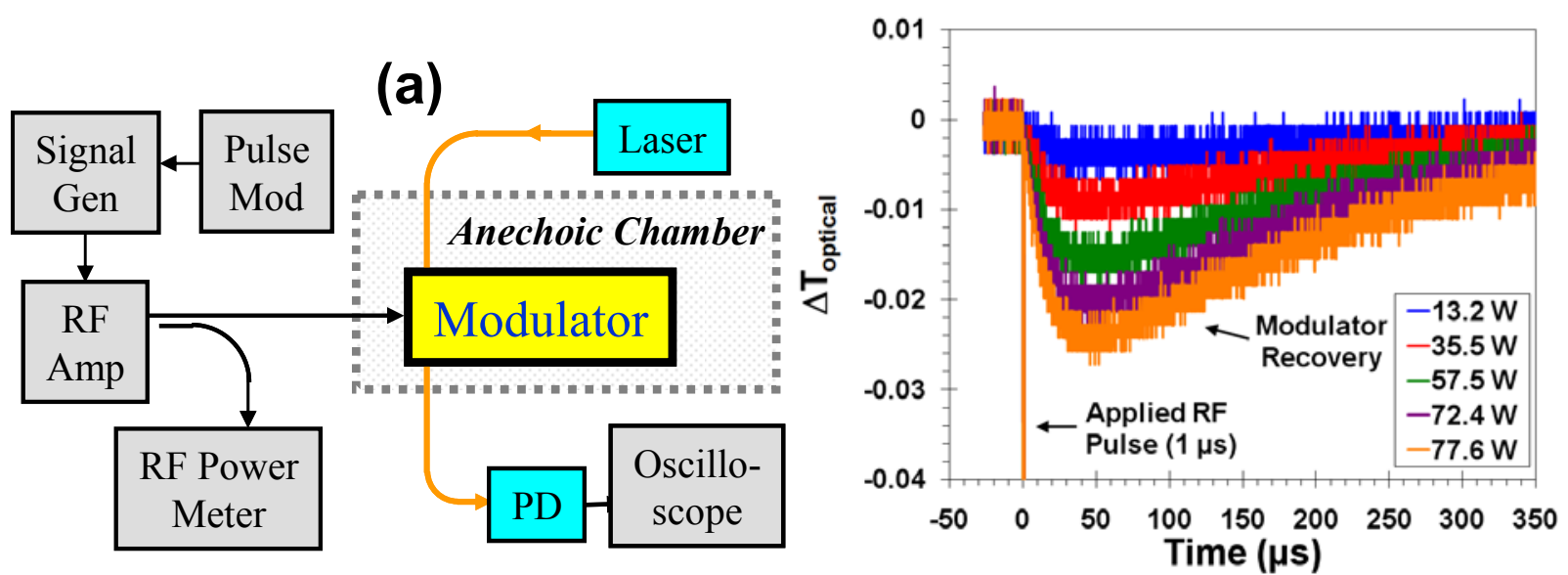

Fig. 1. Photonic link test setup (a), and modulator disruption resulting from a single RF pulse (b).

The single-pulse response for a $1 \mu$ s pulse width and various pulse peak powers is shown in Fig. 1(b), where $T_{\text {optical }}$ is the normalized optical transmission of the modulator $\left(0 \leq T_{\text {optical }} \leq 1\right)$. Following the initial pulse, the modulator response exhibited a clear "after-pulse" that recovered with a 1/e time constant of approximately $300 \mu \mathrm{s}$. At powers greater than a few Watts, this observed change in modulator 
transmission $\Delta T_{\text {optical }}$ was sufficient to substantially disrupt an analog photonic link. The peak change in transmission during the after-pulse was observed to increase linearly with peak pulse power and with pulse width, as shown in Fig. 2(a). This implies that the modulator disruption depended primarily on the RF pulse energy, and along with the $300 \mu$ s time scale suggests RF heating as a probable cause.

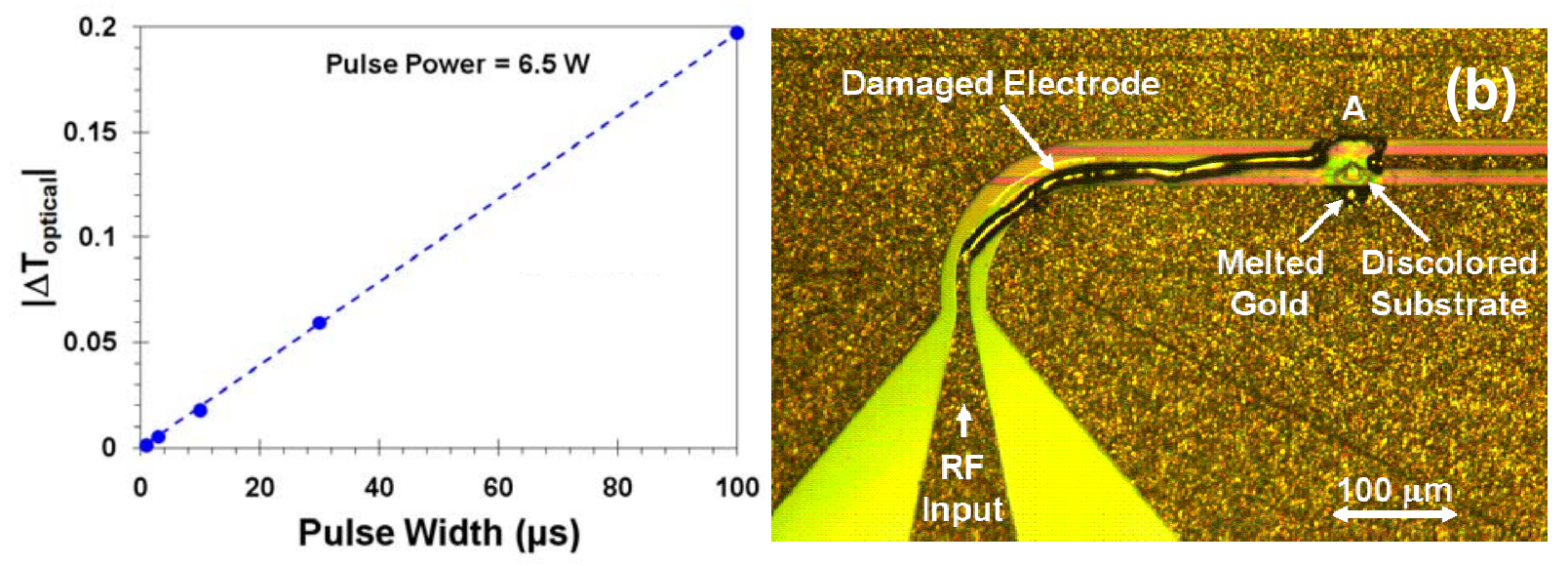

Fig. 2. Link disruption as a function of RF pulse width (a), and damaged modulator electrodes after repetitive RF pulsing at $200 \mathrm{~W}$ peak power, $40 \mu$ s pulse width (b).

The modulator continued to function without noticeable damage after a 10000-pulse burst at $200 \mathrm{~W}$ peak power, $20 \mu$ s width, $2.5 \mathrm{GHz}$ center frequency, and $1 \mathrm{kHz}$ repetition frequency. When the pulse width was increased to $40 \mu \mathrm{s}$, however, the coplanar RF electrode of the modulator was permanently destroyed, as shown in Fig. 2(b). A small portion of the central gold strip was destroyed by electrical breakdown at point "A," which left some gold re-distributed as an electrical bridge to the upper electrode. The portion of central electrode closest the RF input was then partially-melted and separated from the substrate surface due to enhanced Joule heating from the electrical short. That dielectric breakdown would occur at $200 \mathrm{~W}$ is not unreasonable, due to the roughly $11 \mathrm{MV} / \mathrm{m}$ peak electric field across the 13 $\mu \mathrm{m}$ electrode gap. Increasing the electrode gap may help to alleviate this problem.

\section{Conclusion}

These results demonstrate the ability of a commercial $\mathrm{LiNbO}_{3} \mathrm{EO}$ modulator to withstand damage from direct pulsed RF input up to $200 \mathrm{~W}$. However, short-term disruption occurred at a few Watts, which suggests a need for improved modulator thermal design.

\section{Acknowledgements}

The authors wish to acknowledge helpful technical discussions with T. Horton and G. K. Gopalarkrishnan.

\section{References}

[1] M. Abrams, "The Dawn of the E-Bomb," IEEE Spectrum, pp. 24-30, Nov. 2003.

[2] D. A. Fulghum, "Light Boosts Destructive Power of Microwave," Aviation Week and Space Technology, Jan. 21, 2007.

[3] R. C. J. Hsu et al, "All-Dielectric Wireless Receiver," IEEE/MTT-S International Microwave Symposium, pp. 221-224, June 2007. 